\title{
Synergistic Effect of Some Synthetic Peptides and Antibiotics on Efflux Pump of Escherichia coli
}

\author{
Basant $^{1 *}$, Rekha Panwar ${ }^{1}$, Parma Ram Gorachiya ${ }^{2}$, Brij Nandan Shringi ${ }^{1}$, \\ Sunil Maherchandani ${ }^{1}$ and Amit Kumar Pandey ${ }^{3}$
}

\author{
${ }^{1}$ Department of Veterinary Microbiology and Biotechnology, ${ }^{2}$ Department of Livestock \\ Products Technology, ${ }^{3}$ Department of Veterinary Biochemistry, University of Veterinary and \\ Animal Sciences, Bikaner (Rajasthan), India
}

*Corresponding author

\begin{tabular}{|l|}
\hline Ke y w or d s \\
Escherichia coli, \\
Efflux pump, SG-15 \\
and GG-15
\end{tabular}

\begin{abstract}
A B S T R A C T
Expression of efflux pumps and extrusion of antibiotics from bacterial cell through them is one of important innate causes of antimicrobial resistance. The present study aimed to design synthetic peptides as efflux pump inhibitors. Two synthetic peptides SG-15 and GG-15 were designed on the basis of protein sequence and structure of AcrAB-TolC a commonly expressed efflux pump of Escherichia coli (E. coli). SG-15 was designed as inner membrane protein (AcrB) blocker and GG-15 was designed as outer membrane protein (TolC) blocker. The designed inhibitors were custom synthesized. The synergistic effect of these peptides with three antibiotics namely; Ampicillin, Gentamicin and Cephalothin was evaluated through minimum inhibitory concentration of antibiotics alone and with peptides on $10 \mathrm{E}$. coli isolates obtained from mastitic milk samples of cattle. On testing of synergistic effect SG-15 has given better synergistic effect with all the three antibiotics than GG-15 against E. coli but, the extent of effect varied between different antibiotics. Combination of Ampicillin and GG-15 did not show synergistic effect. Interestingly, there was significant synergistic effect on combination of both the peptides with these antibiotics. The designed peptides have shown potent efflux inhibition activity, possibly by blocking the channel present in commonly expressed efflux pump, AcrAB-TolC of E. coli. These peptides have great potential for their synergistic use with antibiotics to reduce efflux pumps mediated antibiotic resistance.
\end{abstract}

\section{Introduction}

Antimicrobial resistance in bacterial pathogen is a global health threat and need efforts to improve this worldwide challenge associated with high morbidity and mortality (Velez et al., 2016). Resistance of important bacterial pathogens to common antimicrobial therapies and emergence of multidrug-resistant bacteria are increasing at an alarming rate (Akova,
2016). The declining effectiveness of antibiotics imposes potentially large health and economic burdens on societies and antibiotic resistance is the next great global challenge significant action to combat it is required.

The use of antibiotics in food animals play a major role in human health, as antibioticresistant bacteria can be transmitted between 
humans and animals through contact, food products and from the environment (Landers et al., 2012). New Delhi Metallo $\beta$ Lactamase-1 (NDM-1) and Expanded Spectrum $\beta$-Lactamases (ESBL) producing gram-negative bacteria (Ghatak et al., 2013) isolated in milk samples obtained from cattle with mastitis have been reported (Eisenberger et al., 2018). Vancomycin-resistant Staphylococcus aureus (VRSA) strains in samples obtained from surgical site (Bhattacharyya et al., 2016) and multidrug resistant $E$. coli was also isolated from the milk sample with clinical mastitis (Todorvic et al., 2018).

Escherichia coli (E. coli), is a common universal inhabitant of gastrointestinal tract and can act as a commensal or a pathogen, being commensal bacteria it is always exposed to antibiotic stress when an individual is treated with an antibiotic such exposures also increase the chances of $E$. coli to become an MDR. Most E. coli strains are harmless, but some pathogenic strains (enteropathogenic E. coli) can cause bloody diarrhea, gastroenteritis, dysentery and urinary tract infections in humans and animals (Levine, 1987).The occurrence of multidrug resistant bacteria is a serious problem in the treatment of bacterial infections (Bolhuis et al., 1997). Increased use of antibiotics has also raised their involvement in transfer of resistance genes to gut bacteria (Van et al., 2001). Therefore, it is important to study resistance profiles of $E$. coli. The prevalence of resistance in commensal $E$. coli is an indicator for the selective pressure by antibiotics used and resistance to be expected in commensal bacteria.

The prevalence of resistance in commensal $E$. coli is a good indicator for the selective pressure by antibiotics used and resistance problem to be expected in pathogenic bacteria (Salehi and Bonab, 2006).
Mechanism of antibiotic resistance includes pumping the applied antibiotic out of bacterial cell wall by efflux pumps (Webber and Piddock, 2003). These pumps are proteinaceous transporters found in prokaryotic as well as eukaryotic cells for performing various physiological functions.

Efflux pumps of efflux superfamilies such as MFS, MATE, SMR and RND are localized in the cytoplasmic membrane and derive energy for extruding of various substrates by the proton motive forces. Among the efflux pumps, only $\mathrm{ABC}$ transporters derive energy by ATP hydrolysis (Sun et al., 2014).

Several drugs have been tried to inhibit the mechanism of such pumps including Phenylalanyl Arginyl $\beta$-naphthylamide $(\mathrm{PA} \beta \mathrm{N})$, globomycin, carbonyl cyanide $\mathrm{m}$ chlorophenylhydrazone (CCCP) and quinolones (Pages et al., 2005). Peptides for blocking of efflux pumps, can be easily designed, synthesized and modified suitably and has attracted attention of several researchers (Poulsen and Deber, 2012; Lamers et al., 2013). The current research aims at screening of newer synthetic peptides for blocking of bacterial efflux pumps.

Synthetic peptides are compounds where multiple amino acids are linked via amide bonds, also known as peptide bonds; these peptides are chemically synthesized by the condensation reaction of carboxyl group of one amino acid to the amino group of another. Synthetic peptides are most widely used to study the relationship between structure and activity of biologically active protein and efflux pumps are protein structure.

A specific advantage is that they can be generated as exact copies of protein fragments as well as in diverse chemical modifications according to the need (Groß et al., 2016). Designing of synthetic peptides against efflux 
pumps can be done by several computational software such as, Peptide Builder (Tien et al., 2013), NHLBI- Ab Designer (Pisitkun et al., 2011) and pepsequencer (Schutkowski et al., 2005) named a few. The efficacy of such peptide along with antibiotics can be checked by the determination of MIC by broth dilution method (Wiegand et al., 2008).

Keeping in view the need to search suitable peptides administered along with antibiotics to block the efflux pumps helping antibiotics to function properly and to prevent economic loss due to futile treatment strategies against serious bacterial infections in animals, the present research studies has been conducted.

\section{Materials and Methods}

\section{Sampling}

In the present investigation out of 28 milk sample 10 isolates of Escherichia coli from mastitis milk of cattle were used. The samples were collected from the RAJUVAS clinics. The samples were collected aseptically and placed in sterile container, taking all precautions to avoid contamination.

\section{Isolation and species level conformation}

The procedure for isolation and identification of bacterial culture was followed as per the standard protocols (Carter et al., 1994). For primary cultivation each isolate was streaked on MacConkey agar plates in primary, secondary, and tertiary fashion in order to obtain isolated colonies of bacteria. These petri plates were incubated for $24 \mathrm{hrs}$ at $37^{\circ} \mathrm{C}$.

After the revival organism isolated colonies were further streaked on to Eosin Methylene Blue (EMB) agar (Edward and Ewing 1986). Besides this MALDI-TOF MS were used for species level conformation also used as per the method described by Singhal et al., 2015.

\section{Ethidium bromide-agar (Et-br) cartwheel method for evaluation of efflux activity}

The test was performed as per the method of Martins et al., 2013. All isolates were grown in $5 \mathrm{ml}$ of nutrient broth and adjusted to 0.5 McFarland with PBS. Trypton soya agar plates containing Et-br concentrations ranging from 0.5 to $2.5 \mathrm{mg} / \mathrm{l}$ were prepared on the same day of the experiment and protected from light by covering with aluminium foil. The plates were then divided into as many as 8 sectors by radial lines (cartwheel pattern).

The isolates were inoculated in sterile $5 \mathrm{ml}$ nutrient broth, incubated for $18 \mathrm{hr}$ at $37^{\circ} \mathrm{C}$ and then the opacity was adjusted to 0.5 McFarland opacity standards with Normal saline solution were swabbed on Et-br agar plates starting from the centre of the plate and spreading towards the edges. The swabbed Etbr agar plates were then incubated at $37^{\circ} \mathrm{C}$ for $16 \mathrm{~h}$ and examined under UV transilluminator and photographed. The minimum concentration of Et-br that produced fluorescence was recorded. For confirmation of efflux activity in isolates this experiment was further repeated with adding of Carbonyl Cyanide m-Chlorophenylhydrazone (CCCP), is a known efflux pump inhibitor acts by inhibition of proton motive forces by was mixed with Et-br in TSA.

\section{Designing of synthetic peptides}

The peptides were designed with help of software AbDesigner and then docking of these peptides and efflux pump protein of AcrAB-TolC complex is done with help of a computational software pepATTRACT 2.0.

In the present investigation we used these peptide sequences to calculate MIC and MBC with selected antibiotics against $E$. coli by which activity of these peptides have been determined as efflux pump inhibitor. 
Determination of minimum inhibitory concentration (MIC) and Minimum bactericidal concentration (MBC)

The MIC is defined as the lowest concentration of antibiotics that inhibit the growth of microbes whereas MBC is defined as the lowest concentration of the antibiotic that completely kill microorganism thereby they fail to grow on subsequent sub-cultured on to antibiotic-free media. MIC and MBC were determined for the antibiotic alone or in combination with designed Peptides and CCCP separately and effects on lowering of MIC of antibiotics were compared.

\section{Results and Discussion}

\section{Isolation and identification of $E$. coli}

A total 10 isolates of E. coli were isolated from 28 milk samples of cattle with clinical mastitis on the basis of cultural characteristics and biochemical tests. $E$. coli isolates revealed characteristic rose pink colonies (lactose fermenting type) on MacConkey agar plates.

Pink colonies were further streaked on Eosin Methylene blue (EMB) agar on which all ten isolates of $E$. coli produced greenish metallic sheen colonies on EMB agar and after that the MALDI-TOF MS used for species level conformation.

\section{Determination of efflux activity of $E$. coli isolates}

Efflux activity of E. coli isolates was determined by Ethidium bromide (Et-br) cartwheel method (Martins et al., 2013) with slight modifications. Et-br is most commonly used dye for detection of efflux activity in the bacterial strains because Et-br is most common substrate for all efflux pumps. The results of this method presented in the form of intensity of florescence given by the isolates which were interpreted as "-", “+”, “++", "+++" and " $++++"$.

\section{Designing of synthetic peptides}

Synthetic peptides have been designed on the basis of amino acid sequence and structure of efflux pump protein AcrAB-TolC, which is tripartite pump protein highly expressed for efflux activity in $E$. coli. It was done by help of a software namely AbDesigner. Designing of two 15 amino acid containing peptides one for inner membrane protein inhibition and another for outer membrane protein inhibition of pump protein.

The 15 amino acid sequences of peptides were taken from the sequence of efflux pump protein (i) from 807- 821 of AcrB protein and (ii) from 271-285 of TolC protein comprising. Designed peptides were synthesized from BioChem Group Labs. Designing was similar to investigation of Bellmann-Sickert et al., 2013; Poulsen et al., 2012.

Comparison of MIC values of selected antibiotics alone and in synergistically with synthetic peptides

A total three antibiotics were selected namely: Gentamicin, Cephalothin and Ampicillin. Gentamicin, which interrupting protein synthesis and reported as substrate for efflux pump of E. coli. Cephalothin, a beta-lactam antibiotic and Ampicillin is amino penicillin also a member of beta-lactam antibiotics acts by inhibition of cell wall synthesis.

Beta-lactam antibiotics are considered as one of the important substrate extruded by efflux pump of E. coli (Anes et al., 2015). CCCP was used as standard efflux pump inhibitor in this experiment so that effects of peptides were compared with it. In present study two synthetic peptides, SG-15 and GG-15 with 
three antibiotics (Gentamicin, Cephalothin and Ampicillin) were evaluated for their synergistic as well as efflux pump inhibitory activity against four E. coli isolates obtained from clinical samples. The MIC and MBC of all these antibiotics were determined in the presence and absence of synthetic peptides.

The MIC values of Gentamicin when tested with E. coli ranged between $4-8 \mu \mathrm{g} / \mathrm{ml}$ respectively. When Gentamicin used in combination with peptide SG-15, MIC value were 4-8 fold decreased and when Gentamicin used in combination with peptide GG-15 there was 2 fold decrease in MIC whereas on combining of both the peptides i.e. SG-15 and GG-15, there was significant decrease in MIC value of 8-16 fold. On comparison of effect of peptides with $\mathrm{CCCP}$ results were almost similar. These results were quite similar to results of (Coutinho et $a l ., 2008)$. They have used Chlorpromazine and Mentha arvensis to reduce MIC of Gentamicin and the MIC values were reduced to $1 / 16$.

The MIC values of Cephalothin when tested with E. coli ranged between 16-32 $\mu \mathrm{g} / \mathrm{ml}$ respectively. When Cephalothin used in combination with peptide SG-15, MIC value were 8-16 fold decreased and when Cephalothin used in combination with peptide GG-15 there was 2-4 fold decrease in MIC whereas on combining of both the peptides i.e. SG-15 and GG-15, there was significant decrease in MIC value of 16-32 fold. On comparison of effect of peptides with CCCP results were almost similar.

The MIC values of ampicillin when tested with E. coli ranged between 4-16 $\mu \mathrm{g} / \mathrm{ml}$ respectively. When ampicillin used in combination with peptide SG-15, MIC value were 2-4 fold decreased and when ampicillin used in combination with peptide GG-15 there was no change in MIC whereas on combining of both the peptides i.e. SG-15 and GG-15, there was 2-4 fold decrease similar to SG-15 which means there was no effect of GG-15 (outer membrane protein blocker). On comparison of effect of peptides with CCCP results were almost similar.

The MBC values of gentamicin when tested with E. coli ranged between 8-32 $\mu \mathrm{g} / \mathrm{ml}$ respectively. When gentamicin used in combination with peptide SG-15, MBC value were 2 fold decreased and when gentamicin used in combination with peptide GG-15 there was no change in MBC values whereas on combining of both the peptides i.e. SG-15 and GG-15, there was only 2 fold decrease in $\mathrm{MBC}$ value. It means only SG-15 (inner membrane protein blocker) was effective to reduce $\mathrm{MBC}$ of gentamicin. On comparison of effect of peptides with CCCP results were almost similar.

The MBC values of cephalothin when tested with E. coli ranged between $64-256 \mu \mathrm{g} / \mathrm{ml}$ respectively. When cephalothin used in combination with peptide SG-15, MBC value were 8-32 fold decreased and when cephalothin used in combination with peptide GG-15 there was 2-8 fold decrease in MBC values whereas on combining of both the peptides i.e. SG-15 and GG-15, there was 832 fold decrease in MBC value. On comparison of effect of peptides with CCCP results were almost similar.

The MBC values of ampicillin when tested with E. coli ranged between 16-64 $\mu \mathrm{g} / \mathrm{ml}$ respectively. When ampicillin used in combination with peptide SG-15, MBC value were 2-4 fold decreased and when ampicillin used in combination with peptide GG-15 there was no change in $\mathrm{MBC}$ values whereas on combining of both the peptides i.e. SG-15 and GG-15, there was only 2 fold decrease in $\mathrm{MBC}$ value. It means only SG-15 (inner membrane protein blocker) was effective to 
reduce $\mathrm{MBC}$ of ampicillin. This may be due to involvement of only inner membrane protein AcrB in extrusion of antibiotic which is involved by taking antibiotics or other substrate in its binding pocket and extrude it by peristaltic mechanism (Edward et al., 2003) On comparison of effect of peptides with CCCP results were similar except for 4 isolates.

All the results of MIC and MBC were suggesting the synergistic effect of designed synthetic peptides and antibiotics were quite similar to synergistic effect antibiotics and CCCP that means the designed peptides were successfully worked as an efflux pump inhibitor by blocking of pump protein.

SG-15 was more effective as compared to GG-15 this may be due to AcrB acts by selection of substrate for pump and binding of substrate in pocket form structure and SG-15 was designed as inner membrane protein blocker and blocking of AcrB was more effective than blocking of TolC.

Efficacies of synthetic peptides as well as CCCP were differing for different antibiotics it may be due to positive or negative interaction of antibiotics and these efflux pump inhibitors and this interaction may be based on structure of antibiotic or nature of antibiotic.

Peptides were more efficient in terms of concentration required as peptides were shown their effect at $1 \mu \mathrm{g} / \mathrm{ml}$ whereas CCCP were effective at $10 \mu \mathrm{g} / \mathrm{ml}$ dissolved in water and DMSO respectively.

Peptides are considered good therapeutic agents because they are having high safety level, tolerability, predictable metabolism and standard synthetic protocols (Fosgerau and Hoffmann, 2015).
There are several mechanisms behind emergence of antibiotic resistance in microbes. It can either be inherited/acquired or non-inheritant/ adaptive mechanisms. Adaptive mechanisms include expression of efflux pumps, reduced number of porins and other cell surface modifications created by stress of low level antibiotics (Bhattacharya et al., 2016). Antimicrobial resistance to multiple antimicrobial agents (Multi drug resistance) can be because of enhanced expression of efflux pumps regulated by various genes. These efflux pumps reported more in gram negative bacteria so there is urgent need to discover new therapeutic agents which act as efflux pump inhibitor and can be used in combination with existing antibiotics (Anes et al., 2015). Being gram negative and ubiquitous in nature, with ability to cause diseases and resistant to a host of antibiotics, E. coli is one of the sentinel organisms being used to study antimicrobial resistance.

Efflux pump expression in E. coli infections is one of the main virulence determinants, which significantly increases bacterial resistance to antibiotics and innate host defenses (Akova, 2016). For the present study two synthetic peptides were designed for commonly expressed efflux pump (AcrABTolC) of E. coli and synthesized from BioChem Group Labs. Efficacies of these peptides were tested on $E$. coli isolated from cattle milk with clinical mastitis. Isolates were studied for their efflux activity in the presence of efflux pump inhibitor, further two designed synthetic peptides were evaluated for their efflux pump inhibition ability by determining the MIC and $\mathrm{MBC}$ of three selected antibiotics- Gentamicin, Cephalothin and Ampicillin alone and in combination with designed peptides and CCCP. 
Table.1 Intensity of Florescence given by E. coli isolate on Et-br plates

\begin{tabular}{|c|c|c|c|c|c|c|c|c|c|c|}
\hline \multirow[t]{2}{*}{ Isolates } & \multicolumn{2}{|c|}{$0.5 \mathrm{mg} / \mathrm{l}$} & \multicolumn{2}{|c|}{$1.0 \mathrm{mg} / \mathrm{l}$} & \multicolumn{2}{|c|}{$1.5 \mathrm{mg} / \mathrm{l}$} & \multicolumn{2}{|c|}{$2.0 \mathrm{mg} / \mathrm{l}$} & \multicolumn{2}{|c|}{$2.5 \mathrm{mg} / \mathrm{l}$} \\
\hline & $\begin{array}{l}\text { Alone } \\
\text { Et-br }\end{array}$ & $\begin{array}{l}\text { With } \\
\text { CCCP }\end{array}$ & $\begin{array}{l}\text { Alone } \\
\text { Et-br }\end{array}$ & $\begin{array}{l}\text { With } \\
\text { CCCP }\end{array}$ & $\begin{array}{l}\text { Alone } \\
\text { Et-br }\end{array}$ & $\begin{array}{l}\text { With } \\
\text { CCCP }\end{array}$ & $\begin{array}{l}\text { Alone } \\
\text { Et-br }\end{array}$ & $\begin{array}{l}\text { With } \\
\text { CCCP }\end{array}$ & $\begin{array}{l}\text { Alone } \\
\text { Et-br }\end{array}$ & $\begin{array}{l}\text { With } \\
\text { CCCP }\end{array}$ \\
\hline BG-1 & + & - & + & - & ++ & + & ++ & + & +++ & ++ \\
\hline BG-2 & - & - & - & - & + & - & + & + & ++ & + \\
\hline BG-3 & + & - & + & - & ++ & + & +++ & + & ++++ & ++ \\
\hline BG-4 & + & - & + & + & + & - & ++ & + & ++++ & +++ \\
\hline BG-5 & + & - & + & - & + & + & ++ & + & +++ & ++ \\
\hline BG-6 & - & - & + & - & + & + & ++ & ++ & +++ & ++ \\
\hline BG-7 & - & - & + & - & + & + & ++ & + & ++ & + \\
\hline BG-8 & + & - & + & + & + & + & +++ & ++ & +++ & ++ \\
\hline BG-9 & - & - & + & + & + & + & ++ & + & +++ & ++ \\
\hline BG-10 & + & - & - & - & + & + & + & + & ++ & + \\
\hline
\end{tabular}

- no florescence, + mild florescence, ++ moderate florescence, +++ strong florescence and

++++ strongest florescence

The effects of peptides in lowering the MIC and $\mathrm{MBC}$ values of antibiotics were compared with effect of CCCP. All these 10 isolates were tested for efflux activity by the Cartwheel method with addition of CCCP. All the $E$. coli isolates produced varying degree of fluorescence at different concentration of Et-br.

By the addition of CCCP into Et-br plates resulted in reduced intensity of fluorescence compared to plates containing only Et-br. After the confirmation of efflux activity in isolates efficacy of designed peptides were evaluated by determination of MIC and MBC of three selected antibiotics viz. Gentamicin, Cephalothin and Ampicillin alone and in combination with peptides. On comparing the MIC and MBC of Gentamicin alone and in combination with peptides and CCCP against E. coli isolates when Gentamicin used in combination with peptide SG-15, MIC value were 4-8 fold decreased whereas MBC value were decreased by 2 fold and when Gentamicin used in combination with peptide GG-15 there was 2 fold decrease in MIC whereas no change in $\mathrm{MBC}$ value by this combination.
On combining of both the peptides i.e. SG-15 and GG-15, there was significant decrease in MIC value of 8-16 folds and 2 fold decreases in MBC. Cephalothin in combination with both the peptides had a considerably higher effect as there was 8-16 fold decrease in MIC values by combining SG-15 and 8-32 fold decrease in MBC. When cephalothin used in combination with peptide GG-15 there was 24 fold decrease in MIC and 2-8 fold decrease in MBC whereas on combining of both the peptides i.e. SG-15 and GG-15, there was significant decrease in MIC value of 16-32 fold and 8-32 fold decrease in MBC.

When Ampicillin used in combination with peptides had shown least synergistic effect as compared to Cephalothin and Gentamicin as combination of Ampicillin and SG-15 reduced MIC value by 2-4 fold and MBC value by 2-4 fold whereas combination of GG-15 and Gentamicin had shown no changes in MIC and MBC values. This was the only combination that has shown no synergistic effect. It indicated that the effect of an antibiotic in combination with efflux pump inhibitor depends on the nature of the antibiotic which acts as substrate for the 
efflux system of the organism. The current study indicated that all the isolates taken for the study from cattle milk with clinical mastitis had exposed to a large number of antibiotics by showing resistance to more than half of antibiotics and having efflux activity which is major innate cause of antimicrobial resistance. Synthetic peptides SG-15 and GG15 had a variable effect in combination with antibiotics.

All three antibiotics in combination with both the peptides gave a synergistic effect against $E$. coli but, the extent of effect varied between different antibiotics. When peptides were used in combination with Cephalothin gave more synergistic effect which is followed by Gentamicin and Ampicillin whereas Ampicillin and GG-15 combination had given no effect.

Antibiotic resistance is major challenge of $21^{\text {st }}$ century and efflux pumps are one of the important innate causes of antimicrobial resistance so there is urgent need to discover new efflux pump inhibitors which will safer and easy to incorporated with antibiotics than existing efflux pump inhibitors. Peptides are more safe and tolerable compounds and can be easily synthesized. In the present study two synthetic peptides SG-15 and GG-15 were designed for blocking of AcrAB-TolC efflux pump of E. coli. The efficacy of both these peptides was tested on 10 pathogenic E. coli isolates from cattle milk with clinical mastitis.

The three antibiotics i.e. Gentamicin, Cephalothin and Ampicillin in combination with SG-15 gave a synergistic effect against $E$. coli but, the extent of effect varied between different antibiotics. When peptide GG-15 was used in combination with Gentamicin, Cephalothin and Ampicillin there was a synergistic effect against bacteria, but the extent of effect of GG-15 was more with Cephalothin and least with Ampicillin but, there was significant synergistic effect on combination of both the peptides with these antibiotics and was found working at par with established efflux pump blocker named carbonyl cyanide m-chlorophenyl hydrazone (CCCP). The designed peptides have shown potent efflux inhibition activity, possibly by blocking the commonly expressed efflux pump, AcrAB-TolC of E. coli. These peptides can be incorporated with antibiotics to reduce antibiotic resistance due to extrusion by efflux pumps.

\section{References}

Akova, M. (2016). Epidemiology of antimicrobial resistance in bloodstream infections. Virulence, 7(3), 252-266.

Anes, J., McCusker, M. P., Fanning, S., \& Martins, M. (2015). The ins and outs of RND efflux pumps in Escherichia coli. Frontiers in microbiology, 6, 587.

Bellmann-Sickert, K., Stone, T. A., Poulsen, B. E., \&Deber, C. M. (2015). Efflux by small multidrug resistance proteins is inhibited by membrane-interactive helix-stapled peptides. Journal of Biological Chemistry,290(3), 17521759.

Bhattacharya, S., Pal, K., Jain, S., Chatterjee, S. S., \&Konar, J. (2016). Surgical site infection by methicillin resistant staphylococcus aureus-On decline?. Journal of clinical and diagnostic research: JCDR, 10(9), DC32.

Bolhuis, H., van Veen, H. W., Poolman, B., Driessen, A. J., \&Konings, W. N. (1997). Mechanisms of multidrug transporters. FEMS microbiology reviews, 21(1), 55-84.

Carter, M. E., Quinn, P. J., Markey, B., \& Carter, G. R. (1994). Enterobacteriaceae. Clinical veterinary Microbiology, 209-36. Martins, M., McCusker, M. P., Viveiros, M., Couto, I., Fanning, S., Pagès, J. M., \& Amaral, 
L. (2013). A simple method for assessment of MDR bacteria for overexpressed efflux pumps. The Open Microbiology journal, 7, 72.

Coutinho, H. D., Costa, J. G., Lima, E. O., Falcão-Silva, V. S., \& Siqueira-Júnior, J. P. (2008). Enhancement of the antibiotic activity against a multiresistant Escherichia coli by Mentha arvensis L. and chlorpromazine. Chemotherapy, 54(4), 328-330.

Edward, W. Y., McDermott, G., Zgurskaya, H. I., Nikaido, H., \&Koshland, D. E. (2003). Structural basis of multiple drug-binding capacity of the AcrB multidrug efflux pump. Science, 300(5621), 976-980.

Eisenberger, D., Carl, A., Balsliemke, J., Kämpf, P., Nickel, S., Schulze, G., \&Valenza, G. (2018). Molecular characterization of extended-spectrum $\beta$-lactamase-producing Escherichia coli isolates from milk samples of dairy cows with mastitis in Bavaria, Germany. Microbial Drug Resistance, 24(4), 505-510.

Fosgerau, K., \& Hoffmann, T. (2015). Peptide therapeutics: current status and future directions. Drug discovery today, 20(1), 122-128.

Ghatak, S., Singha, A., Sen, A., Guha, C., Ahuja, A., Bhattacharjee, U., and Dey, T. K. (2013). Detection of New D elhi Metallo-beta-Lactamase and ExtendedSpectrum beta-Lactamase Genes in E scherichia coli Isolated from Mastitic Milk Samples. Transboundary and emerging diseases, 60(5), 385-389.

Groß, A., Hashimoto, C., Sticht, H., \& Eichler, J. (2016). Synthetic peptides as protein mimics. Frontiers in bioengineering and biotechnology, 3 , 211.

Lamers, R. P., Cavallari, J. F., \& Burrows, L. L. (2013). The efflux inhibitor phenylalanine-arginine betanaphthylamide $(\mathrm{PA} \beta \mathrm{N})$ permeabilizes the outer membrane of gram-negative bacteria. PLoS One, 8(3), e60666.

Landers, T. F., Cohen, B., Wittum, T. E., \& Larson, E. L. (2012). A review of antibiotic use in food animals: perspective, policy, and potential. Public health reports, 127(1), 4-22.

Levine, M. M. (1987). Escherichia coli that cause diarrhea: enterotoxigenic, enteropathogenic, enteroinvasive, enterohemorrhagic, and enteroadherent.

Pagès, J. M., Masi, M., \&Barbe, J. (2005). Inhibitors of efflux pumps in Gramnegative bacteria. Trends in molecular medicine, 11(8), 382-389.

Pisitkun, T., Hoffert, J. D., Saeed, F., \& Knepper, M. A. (2011). NHLBIAbDesigner: an online tool for design of peptide-directed antibodies. American Journal of Physiology-Cell Physiology, 302(1), C154-C164.

Poulsen, B. E., \&Deber, C. M. (2012). Drug efflux by a small multidrug resistance protein is inhibited by a transmembrane peptide. Antimicrobial agents and chemotherapy, 56(7), 3911-3916.

Poulsen, B. E., \&Deber, C. M. (2012). Drug efflux by a small multidrug resistance protein is inhibited by a transmembrane peptide. Antimicrobial agents and chemotherapy, 56(7), 3911-3916.

Salehi, T.Z. and Bonab, S.F. (2006). Antibiotics susceptibility pattern of Escherichia coli strains isolated from chickens with coli septicemia in Tabriz Province, Iran. Int. J. of Poultry Sci., 5: 677-684.

Schutkowski, M., Reineke, U., \& Reimer, U. (2005). Peptide arrays for kinase profiling. Chembiochem, 6(3), 513-521.

Singhal, N., Kumar, M., Kanaujia, P. K. \&Virdi, J. S. (2015). MALDI-TOF mass spectrometry: an emerging 
technology for microbial identification and diagnosis. Frontiers in microbiology, 6, 791.

Sun, J., Deng, Z., \& Yan, A. (2014). Bacterial multidrug efflux pumps: mechanisms, physiology and pharmacological exploitations. Biochemical and biophysical research communications, 453(2), 254-267.

Tien, M. Z., Meyer, A. G., Sydykova, D. K., Spielman, S. J., \& Wilke, C. O. (2013). Maximum allowed solvent accessibilites of residues in proteins. PloS one, 8(11), e80635.

Todorović, D., Velhner, M., Grego, E., Vidanović, D., Milanov, D., Krnjaić, D., \&Kehrenberg, C. (2018). Molecular Characterization of Multidrug-Resistant Escherichia coli Isolates from Bovine Clinical Mastitis and Pigs in the Vojvodina Province, Serbia. Microbial Drug Resistance, 24(1), 95-103.
Driessen, C. A. G. G., \& Stobberingh, E. E. (2001). Antibiotic resistance of faecal Escherichia coli in poultry, poultry farmers and poultry slaughterers. Journal of Antimicrobial Chemotherapy, 47(6), 763-771.

Velez, R., \&Sloand, E. (2016). Combating antibiotic resistance, mitigating future threats and ongoing initiatives. Journal of clinical nursing, 25(13-14), 18861889.

Webber, M. A., \& Piddock, L. J. V. (2003). The importance of efflux pumps in bacterial antibiotic resistance. Journal of Antimicrobial Chemotherapy, 51(1), 9-11.

Wiegand, I., Hilpert, K., \& Hancock, R. E. (2008). Agar and broth dilution methods to determine the minimal inhibitory concentration (MIC) of antimicrobial substances. Nature protocols, 3(2), 163.

Van den Bogaard, A. E., London, N.,

\section{How to cite this article:}

Basant, Rekha Panwar, Parma Ram Gorachiya, Brij Nandan Shringi, Sunil Maherchandani and Amit Kumar Pandey. 2020. Synergistic Effect of Some Synthetic Peptides and Antibiotics on Efflux Pump of Escherichia coli. Int.J.Curr.Microbiol.App.Sci. 9(06): 2867-2876. doi: https://doi.org/10.20546/ijcmas.2020.906.347 